American Journal of Applied Sciences 4 (9): 679-685, 2007

ISSN 1546-9239

(C) 2007 Science Publications

\title{
Development of an Axisymmetric Thermal Plume between Vertical Plates
}

\author{
${ }^{1}$ Jamil Zinoubi, ${ }^{2}$ Adel Gammoudi, ${ }^{2}$ Taoufik Naffouti, ${ }^{2}$ Rejeb Ben Maad and ${ }^{2}$ Ali Belghith \\ ${ }^{1}$ Preparatory Institute of the Engineers Studies of Nabeul, Academic Campus \\ El Merazka, 8000 Nabeul, Tunisia \\ ${ }^{2}$ Faculty of Sciences of Tunis, Department of Physics \\ Laboratory of Energizing and Thermal and Mass Transfers, El Manar 2092, Tunis, Tunisia
}

\begin{abstract}
This investigation analyses the turbulent structure of a thermal plume created by a heated disk, evolving between vertical plates. The objective of the study was to understand the development mechanisms of this buoyancy driven flow. The analysis of the average and the fluctuating fields of the temperature as well as the flow visualisation show the existence of three different zones. The first zone of the plume air feeding was characterized by the thermal profiles in three extrema structures. These extrema disappear in the second zone where the profiles present on maximum. In the last zone, the profiles are flattened and self-similar. Thus, the turbulence was fully developed. A flow visualisation show that the vertical channel around the hot disk affects the flow structure plume and causes the appearance of a new zone at the system entrance. This supplementary zone characterizes by the development of the two rotating rolls in the vicinity of the hot source. The skewness and flatness factors of the temperature fluctuations show that the latter have a nearly Gaussian distribution in the most turbulent regions of the flow (in the region where the turbulence was fully developed), but that deviates gradually from the Gaussian distribution in the other region.
\end{abstract}

Key words: Turbulent flow, thermal plume, visualization, skewness and flatness factors, spectral density, cascade concept of Kolmogorov

\section{INTRODUCTION}

Considerable interest has been shown in recent years in the problem of thermal plume development, evolving in an unlimited and in an enclosed environment. Such systems are of practical importance in the environmental problems, to the emission of gas in the atmosphere. They may also be useful in solar heating and ventilating applications for domestic purposes.

Most previous investigators have focussed their attention on the structure of mean and turbulent flow in jet and plume (i.e. in flows driven primarily by buoyancy forces). The theoretical and experimental investigations on thermal plumes profited first by works on jets. However, plumes are essentially governed by buoyancy forces, therefore their fundamental characteristics cannot be deduced from jet investigations. The previous works available on the plume and the warm jets with weak momentum and strong buoyancy at the source $\mathrm{e}^{[1-6]}$.

The flow structure becomes much more complicated for the interaction between a free plume and its physical surrounding arises very frequently in practice. This interaction generates a very complex mechanism $^{[7-10]}$. The plume in enclosures is not independent of the flow at the walls (i.e. Thermosiphon flow). The two flows are obviously coupled in most cases. This coupling introduces a considerable complexity in the analysis of internal flows. The problem of interaction was the subject of only few studies, among which one can mention: the effect of an adjacent vertical surface on plane plume flow ${ }^{[11,12]}$, the interaction between two thermal plumes of equal and also unequal heat ${ }^{[11-14]}$.

The lack of information on the development of an axisymmetric thermal plume between vertical plates motivated the present work. Thermal radiation emitted by the hot disk heats the vertical plates. The pressure drop due to the acceleration of the flow at the wall causes the appearance of thermosiphon effect around the thermal plume. This paper investigates the evolution of the thermal field of an axisymmetric turbulent plume, guided by two vertical plates. This study focussed on understanding the mechanisms of developments of this buoyancy driven flow and the effect of the vertical plates on the flow plume structure.

Experimental apparatus and measurement techniques: The experimental apparatus is shown in Fig. 1. The numbers in the description presented below refer to the part numbers of Fig. 1. The thermal plume is created by a flat disk ${ }^{(1)}$ having a $0.07 \mathrm{~m}$ diameter. The plume source is electrically heated at $300^{\circ} \mathrm{C}$ on the surface by Joule effect. The disk is placed at the entrance of the open ended steel vertical channel ${ }^{(2)}$, which has $0.15 \mathrm{~m}$ spacing and a $0.4 \mathrm{~m}$ height. The system is placed on a frame ${ }^{(3)}$ at $80 \mathrm{~cm}$ above the ground to allow air supply from below. The uniformity of the source surface temperature is obtained by the use

Corresponding Author: Jamil Zinoubi, Preparatory Institute of the Engineers Studies of Nabeul, Academic Campus, El Merazka, 8000 Nabeul, Tunisia, Tel.+216 98213 316; Fax. + 21671560723 
of wire resistors mounted behind the disk. A thermal regulation apparatus kept the temperature of the disk as uniform as possible within a good approximation. $\mathrm{Al}-\mathrm{Cr}$ thermocouples are used to measure the surface temperature of the disk. The difference in temperature between the end and the centre of the disk is less than $5^{\circ} \mathrm{C}$. The vertical channel was thermally insulated by an Armaflex bed, which has a $0.02 \mathrm{~m}$ thickness.

The strong dependence of the flow to the surrounding conditions, require making evolve him inside a quiet atmosphere; so, the experimental device has been placed in an independent closed room ${ }^{(4)}$.

To explore the voltage signals corresponding to the temperature we have used the resistant wire anemometer at constant current ${ }^{(5)}$. This technique adopted for a long time by Doan Kim-Son et al. ${ }^{[15]}$. in natural convection, is based on the principle of the resistance variation of a platinum wire $(7.5 \mu \mathrm{m}$ in diameter).

In order to avoid the disruption of the flow, the probe is introduced vertically, by the system exit, so that its sensible wire was perpendicular to the ascending flow. The wire thermal inertia, where its time constant is the order of $1 \mathrm{~ms}$, introduced not of measure mistake, especially to the weak frequencies met in plumes ${ }^{[12]}$. Mistakes coming from the probe calibration are lower than $1 \%$.

On the other hand, in order to explore thermal voltage signals at every level of the flow, we have used a displacement system ${ }^{(6)}$ of the probe, following two directions. The minimal displacement in the vertical direction is $10^{-3} \mathrm{~m}$, whereas in the horizontal direction is $2.10^{-5} \mathrm{~m}$.

A computer ${ }^{(7)}$ provided of a data acquisition card permits to appropriate of the instantaneous measures all $10 \mathrm{~ms}$ and to record signals for further statistic processing.

The visualization system used, during this study, was constituted of a He-Ne laser ${ }^{(8)}$ with a power of $35 \mathrm{~mW}$ an electric vibrant plate $^{(9)}$, a digital camera ${ }^{(10)}$ and a smoke tracer distributor.

On the vibrant plate is glued a plane mirror of good quality, that receives a horizontal laser beam and it reflects according to the channel vertical axis; the displacement of the plate makes revolve the mirror around a horizontal axis, creating a plane laser sheet ${ }^{(11)}$ on the entire transverse section of the channel. Smoke from incense oil was used as tracer. The smoke tracer is naturally entrained into the flow region without any disruption.

\section{RESULTS AND DISCUSSION}

Flow visualization: In order to clarify the thermal plume evolving between two vertical plates, the flow visualization should be carried out. The flow visualization performed by illuminating the flow by a plane laser sheet is presented in Figs. 2-4. These instantaneous photographs show the behavior of the flow in the studied case (a flow between vertical plates). For these Figs the sowing of the flow takes place all along the channel.

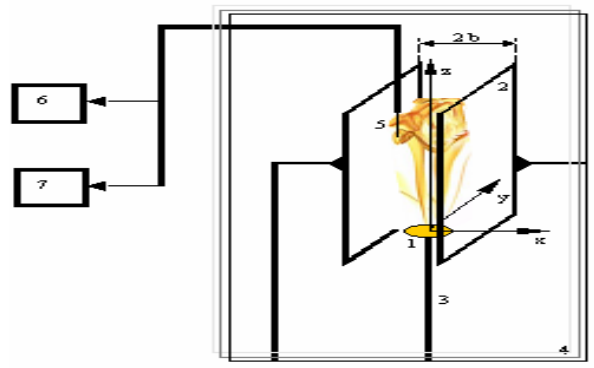

Fig. 1a: Experimental setup

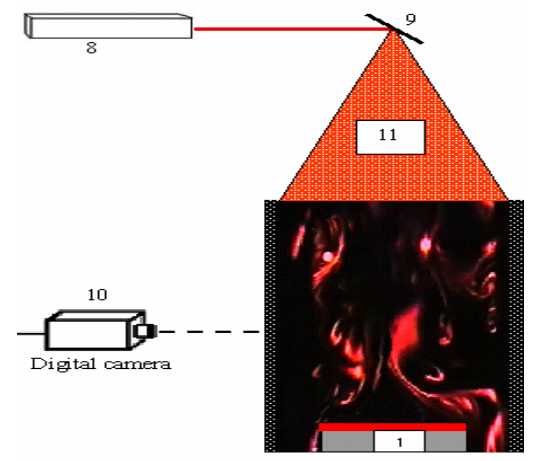

Fig. 1b: Visualization system

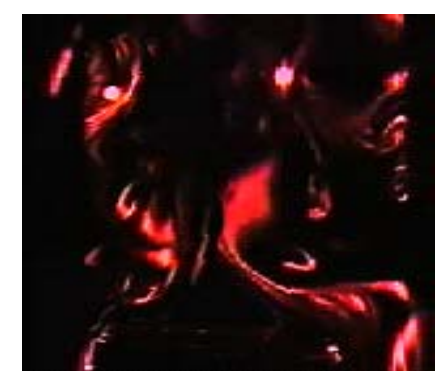

Fig. 2: General view of the flow

Indeed, these Figs. show that the plume and the vertical plates supply themselves regularly in fresh air by the low. The fresh air arriving from the outside, by every side of the hot source is submitted to an 


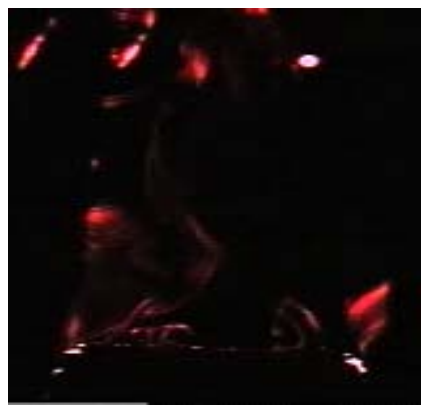

Fig. 3: Exhausts of the left roll

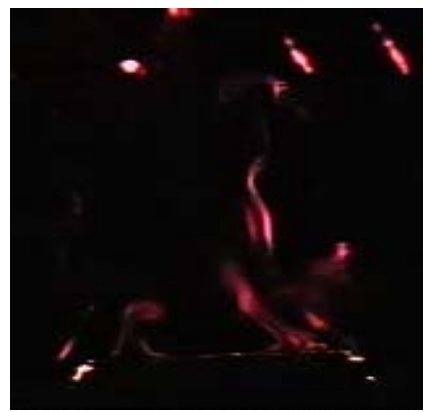

Fig. 4: Exhausts of the right roll

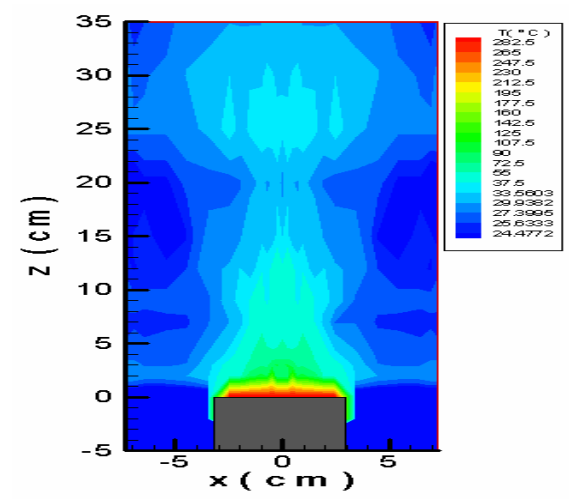

Fig. 5: Isotherms fields of the flow

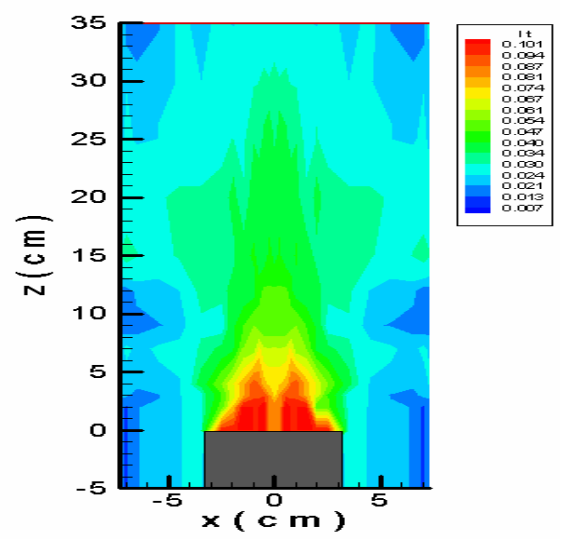

Fig. 6: Iso-values of standard deviations of the flow interaction between the plume and the plates, before pursuing its path directly toward the central part of the flow

In the vicinity of the hot source the instantaneous photos showed, above the hot disk, the development of two rotating rolls symmetrical to the plume axis (Figs. 3 and 4). The formation of these rolls could be explained by the presence of trapped hot air near the source. In fact, the fresh air layers, arriving from the outside, find themselves on the top of the relatively hotter and less dense fluid layers adjacent to the hot surface. This is an unstable fluid stratification that results in a convective motion leading to the formation of a vortex that is created close to the hot source. The trapped fluid continues to turn. When this air mass acquires a sufficient energy, it escapes vertically (Figs. 3 and 4). To replace this fluid, this region is supply again in fresh air that will undergo, the same process. We note the existence of a certain alternation however between the formation and the exhaust of these two rolls. Indeed, these two mechanisms don't occur simultaneously; as one of the rollers escapes, the other forms.

The visualization indicates the existence of three different structure zones of the flow vertical development. Therefore, in the first zone in the vicinity of the hot source $(\mathrm{z} \leq 5 \mathrm{~cm})$, This visualization shows, above the hot disk, the development of two rotating rolls symmetrical to the plume axis. In the intermediary zone $(5 \mathrm{~cm}<\mathrm{z} \leq 15 \mathrm{~cm}$ ), we observe a contraction of the plume and an ascending strong axial flow caused by buoyancy forces. In the third zone $(\mathrm{z}>15 \mathrm{~cm})$, this visualization shows the appearance of new flow behaviour where a small structure occupies all the upper part of the channel. The existence of these turbulent dissipating structures leads to a uniform flow in the channel exit.

Thermal average and fluctuating fields: General view of the flow: The standard deviations of the flow is given by the following expression:

$$
I_{t}=\frac{\sqrt{\overline{T^{\prime 2}}}}{T_{s}-T_{\infty}},
$$

where $T^{\prime}$ is the temperature fluctuation and $T_{s}-T_{\infty}$ is the temperature difference between the source and the ambient medium.

In Figs. 5 and 6 we report the isotherms fields and the iso-values of the standard deviations tracing in a flow symmetry plan. These Figs. reveal the evolution of the flow from the system entry to its exit and show the region occupied by the plume during its ascension and inform us on the most turbulent region. Fig. 6 
shows the existence of the two rotating rolls above the hot source described previously. These Figs. also indicate the existence of three different structure zones of the flow vertical development ${ }^{[7-10]}$.

Vertical evolution of the flow: Flow zones: The axial evolution of the dimensionless extrema mean temperature and the standard deviations of the flow are plotted in Fig. 7. This Fig. indicates several behaviors. In the first zone near the hot source, a strong decrease of the temperature and the standard deviations is observed. From $5 \mathrm{~cm}$ level, a process of behavior change starts; this process characterizes the second zone of the flow. Beyond $15 \mathrm{~cm}$ level the gap between the maximal and the one of the minimal nearly maintains itself at a value constant. It characterizes the third aspect of the flow that occupies the superior part of the channel.

As the flow visualization, the vertical evolution shows the existence of three different zones characterizes the flow vertical development.

Thermal average fields: Figure 8 presented the radial distribution of the dimensionless average temperature of the flow given by:

$$
T=\frac{\bar{T}-T_{\infty}}{T_{s}-T_{\infty}}
$$

where $\bar{T}$ is the mean temperature.

For a lower level $(\mathrm{z} / \mathrm{b}=0.285)$, the flow is strongly affected by the hot disk. Figure 8a shows the existence of three extrema profiles with a minimum on the axis and two symmetrical maxima located on each side of the axis. These temperatures maxima are attributed to an exhaust mechanism of two rotating rolls, similar rolls were observed by A.O.M. Mahmoud et al. ${ }^{[7]}$, whereas average temperature drops falls down, on the plume axis, which is due to a strong fresh air penetration coming from the ambient medium. These profiles show an important transversal gradient of temperature in each side of the maximum. This strong gradient results from the brutal transformation of the ambient air to a hot plume in the region close to the source.

For the second zone $(\mathrm{z} / \mathrm{b}=1.714)$ the structure of three extrema disappears; the temperature profiles present only one maximum on the axis (Fig. 8b). This structure is comparable to free plume one ${ }^{[7-9]}$.

For the third zone $(\mathrm{z} / \mathrm{b}=4.285)$, where the turbulence is fully developed, the temperature profiles become flat with a nil transverse gradient, thus translating the uniformity of the flow temperature (Fig. 8c).
Thermal fluctuating fields:Standard deviations: Like the average temperature field, at smaller heights $(\mathrm{z} / \mathrm{b}=0.285)$, the standard deviations exhibit two axisymmetric maxima, located on each side of the axis (Fig. 9a). These maxima result from the interaction of a convection flow that feeds the center of the plume and the hot air escaping from the rotating rolls. These two maxima correspond to the regions where the interaction between the plume and the ambient air is the most

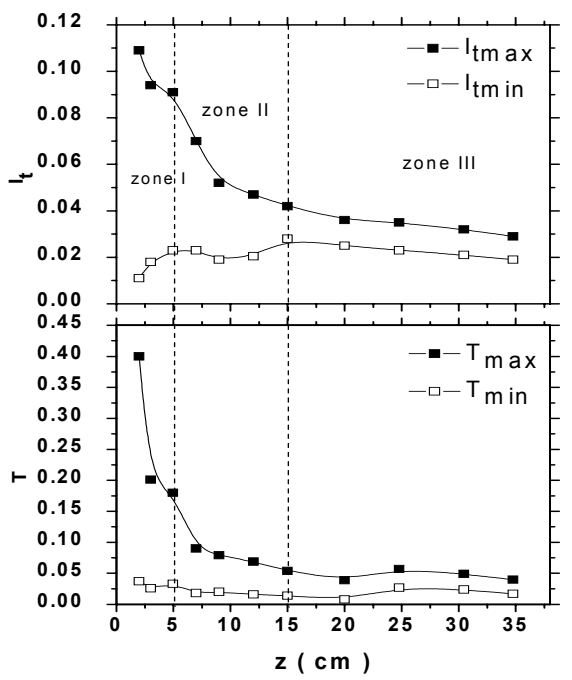

Fig. 7: Axial evolution of the dimensionless extrema mean temperature and the standard deviations of the flow

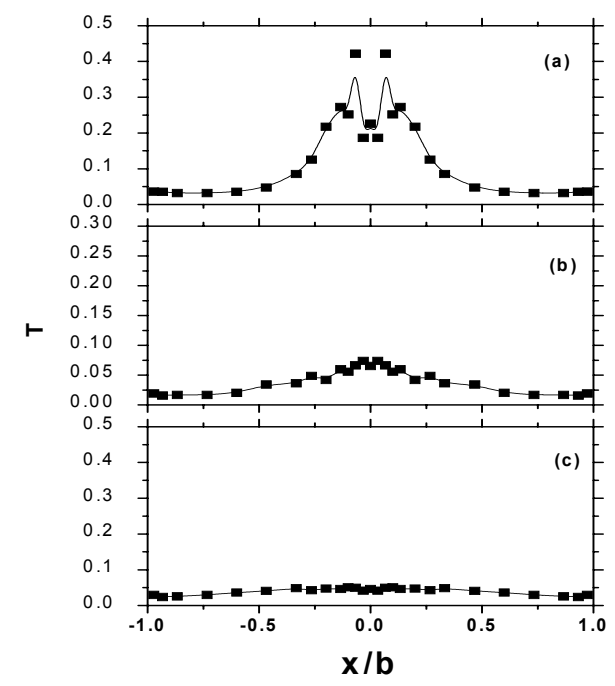

Fig. 8: Radial distribution of the dimensionless average temperature of the flow: (a) $\mathrm{z} / \mathrm{b}=0.285$, (b) $\mathrm{z} / \mathrm{b}=1.714$, (c) $\mathrm{z} / \mathrm{b}=4.285$

important. Near the vertical plates the standard deviations are always low. On the axis, a clear decrease 
is observed, so, the flow is disturbed because it receives directly a flow thread from the ambient that is influenced by the rotating rolls. The homogenization of the turbulence progresses slowly as the height increases (Figs. 9b and 9c).

Skewness and flatness factors: The investigation of the skewness and flatness factors, respectively given by:

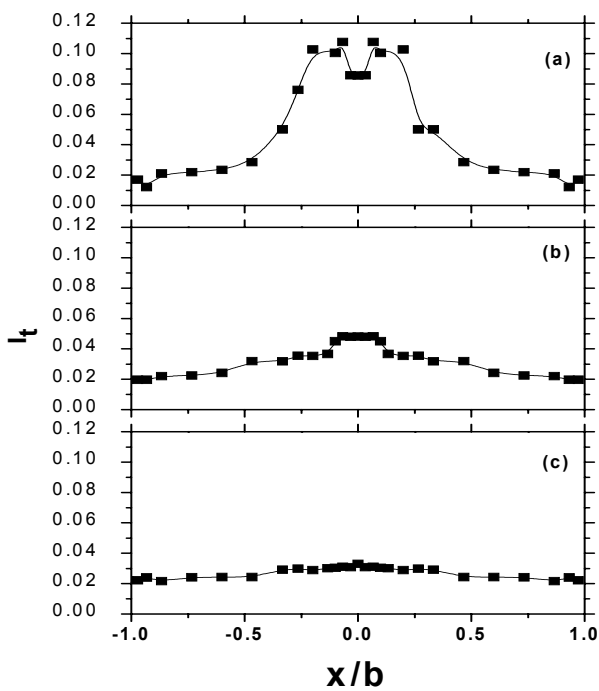

Fig. 9: Radial distribution of the standard deviations of the flow: (a) $z / b=0.285$, (b) $z / b=1.714$, (c) $\mathrm{z} / \mathrm{b}=4.285$

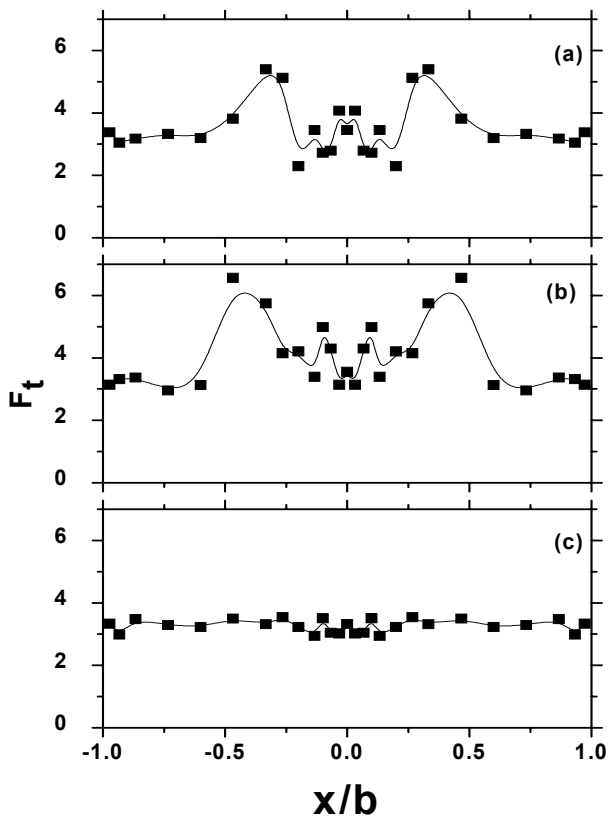

Fig. 10: Radial distribution of the flatness factor: (a) $\mathrm{z} / \mathrm{b}=0.285$, (b) $\mathrm{z} / \mathrm{b}=1.714$, (c) $\mathrm{z} / \mathrm{b}=4.285$

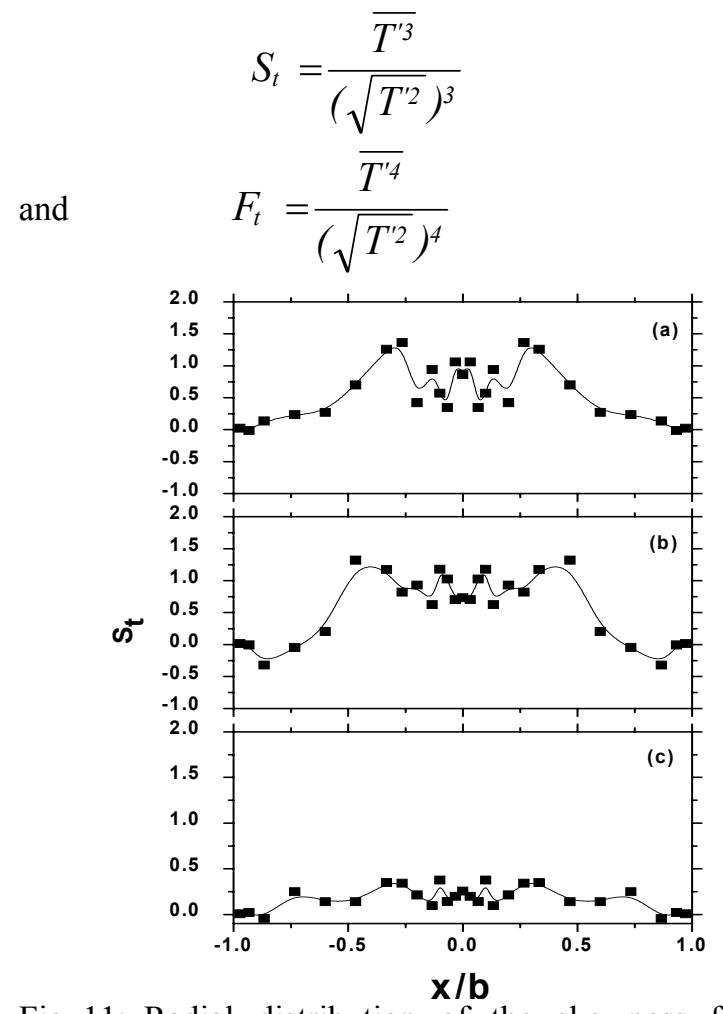

Fig. 11: Radial distribution of the skewness factor:

(a) $\mathrm{z} / \mathrm{b}=0.285$, (b) $\mathrm{z} / \mathrm{b}=1.714$, (c) $\mathrm{z} / \mathrm{b}=4.285$

These parameters allow the comparison of the probability density function of the flow temperature fluctuations and the ideal Gaussian distribution $S_{t}=0$ and $\mathrm{F}_{\mathrm{t}}=3$.

The radial distribution of the skewness and flatness factor are given in Figs. 10 and 11. For the levels $(\mathrm{z} / \mathrm{b}=0.285$ and 1.714$)$, the flatness factor, at every side of the disk edge, approaches the values $F_{t}=3$ and the skewness factor approaches $S_{t}=0$ (Figs. 10a, 10b, $11 \mathrm{a}$ and $11 \mathrm{~b}$ ). Consequently, it is deduced that in this region, the probability density governing the temperature fluctuations in the flow follows approximately the ideal Gaussian distribution. In every side of the axis, the flatness and the skewness factor presents two maxima. These high values of the thermal flatness and the skewness factor correspond to the regions of the flow where the interaction between the plume and the ambient air is the most important $t^{[4]}$. These values would be associated with the intermittency phenomenon ${ }^{[9]}$. The intermittency results from the interaction between the plume flow and the enveloping thermosiphon ${ }^{[9]}$. It provides information about more or less turbulent regions of the flow. It also indicates the degree of penetration of laminar fluid from outside ${ }^{[14]}$. 
At the core of the flow, the skewness factor shows a prevailing of the positive fluctuations characterizing the hot air puffs. The zero value of the skewness factor indicates an equal probability of the presence of the hot air coming from the plume and the ambient air. The negative skewness factor, characterize a strong flow of cold air arising from the ambient media. This air supplies the source of the plume in a region close to its axis and the flow along the hot plates.

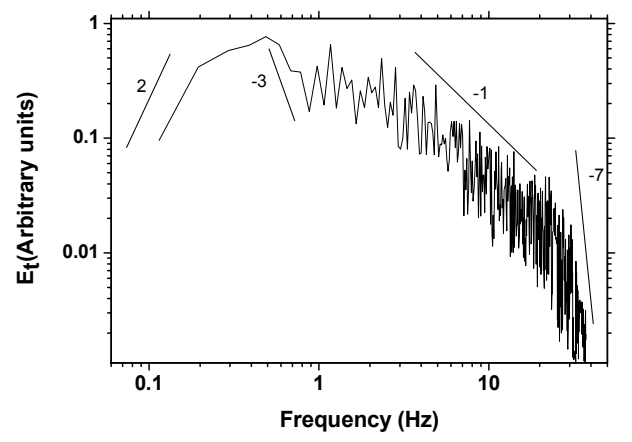

Fig.12: Power spectral density of the temperature fluctuations $-\mathrm{z} / \mathrm{b}=4.28$

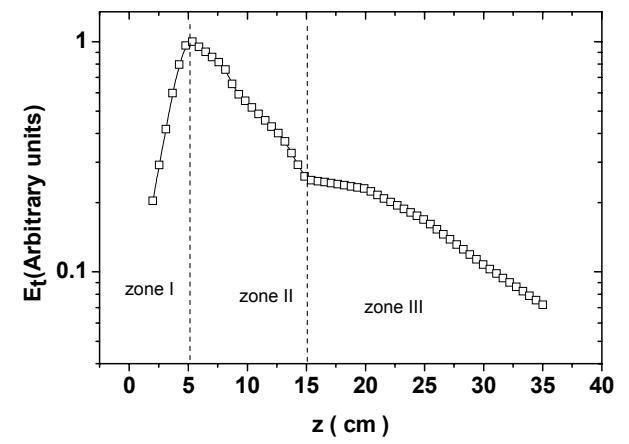

Fig. 13: Vertical evolution of the normalized power spectral density of the temperature fluctuations

For the level $(\mathrm{z} / \mathrm{b}=4.285)$, where the turbulence is fully developed, the flatness and the skewness factor profiles become flat, showing a thermal homogenization; in this region as seen, in Fig. 10c and 11c, the flatness and the skewness factor respectively approaches the value $F_{t}=$ 3 and $S_{t}=0$. Consequently, the distribution of the temperature fluctuations is close to a Gaussian distribution.

Spectral density of the temperature fluctuations: Studying thermal plumes entails the understanding of the phenomenon itself, flow regimes, buoyancy influence and turbulent transport and production phenomena. The understanding of these phenomena can be based on spectral analysis according to the energy cascade concept developed by Kolmogorov. A spectral analysis using Fast Fourier Transformation (FFT) was carried out. The temperature spectral density $\mathrm{E}_{\mathrm{t}}(\mathrm{n})$ investigated at the level $(\mathrm{z} / \mathrm{D}>2.14)$, where the turbulence is fully developed, is standardised under the following from:

$$
\int_{0}^{\infty} E_{t}(n) d n=1
$$

Figure 12 shows the plot of the normalized spectral energy as a function of the frequency (in Log-Log coordinates). A typical spectrum of temperature fluctuations corresponding to an experimental configuration where the height measurement $\mathrm{z} / \mathrm{b}=4.28$. As the frequency increases, i.e. for smaller structures, the normalised energy tends to decrease. This behavior is consistent with the usual cascade scenario toward small length scales.

Different spectral regions can be identified in Fig. 12. The first region is a production region characterized by a law $\mathrm{n}^{2}$, due to turbulent flux. The second region is quite interesting and is characterized by a law $\mathrm{n}^{-3}$, corresponding to a buoyancy region, typical in buoyant free convection flows. Such these behavior has been, already reported by Lumley ${ }^{[16]}$ in a spectral analysis of quasi-inertial turbulent flows in stably stratified environments and by several other authors for round plumes ${ }^{[2,5,17,18]}$. Rather than giving an analytical explanation of this -3 slope, we should emphasize the absence of the classical $-5 / 3$ slope, (although previous studies reported such behavior). We know that the existence of a constant $-5 / 3$ slope region is related to a hypothesis of homogeneity and isotropy. The appearance of a -3 power law region (inertialdiffusive sub range ${ }^{[2]}$ ) may be related with the passage in the measurement volume of large structures ${ }^{[17]}$ generated by the hot source. The $\mathrm{n}^{-1}$ Tchen law appears clearly that related to an important turbulent production. A last region was also identified. This so-called dissipative region, of very fast decay, is consistent with an $\mathrm{n}^{-7}$ law. This kind of law was, proposed from theoretical considerations, by Corrsin ${ }^{[19]}$, in a study of the temperature fluctuation spectra for isotropic turbulent flows.

Figure 13 shows the vertical evolution of the normalized spectral energy. In the first zone near the hot source, this Fig. shows that the normalised energy tends to increase with $\mathrm{z}$. This is due to turbulent flux. From $5 \mathrm{~cm}$ level a decrease process of the spectral energy flow starts. This process characterises transfer region. When approaching the third zone (i.e. from $15 \mathrm{~cm}$ level), this profile changes pace. These structures are responsible for energy transfer modes. Buoyancy effects seem to impose the rate at which potential energy is converted into kinetic energy of the larger scales. Taking over viscous effects (in comparison to 
flows for which energy transfers are driven essentially by turbulent shear), this additionally entails noticeable modifications of the inertial equilibrium range within which the energy of the larger scales is transferred to smaller scales according to the cascade concept of Kolmogorov.

\section{CONCLUSION}

We have described the results of an experimental study that examines the turbulent structure of the thermal plume issued from a heated disk evolving in a semi-enclosed environment.

The visualization of the flow as well as the thermal average and fluctuating fields study have showed that the global structure of the flow is constituted of three different zones; a first zone of instability close to the source $(\mathrm{z} / \mathrm{b} \leq 0.285)$, where the strong plumethermosiphon interaction entailed the apparition of the rotating rolls. A second intermediate zone $(0.285<\mathrm{z} / \mathrm{b}$ $\leq 1.714)$, where develops the interaction flow. A last zone $(\mathrm{z} / \mathrm{b}>1.714)$, where the turbulence is fully developed, that occupies the superior part of the channel.

The skewness and flatness factors show that the law governing the temperature fluctuations approximates the Gaussian law quite well in the region where the turbulence is fully developed and deviates gradually from this law in the other regions. A predominance of the positive fluctuations characterizing puffs of hot air generated by the turbulent rolls was also noticed.

The spectra of temperature fluctuations obtained enabled identifying three distinct spectral regions that correspond to energy transfer mechanisms between scales: a production region due to turbulent flux characterized by $\mathrm{a}^{2} \mathrm{n}^{2}$ law; a buoyancy region characterized by a $\mathrm{n}^{-3}$ law, characteristic of anisotropic turbulence caused by buoyancy that modifies the inertial equilibrium range within which energy of the larger scales is transferred to smaller scales and finally, a dissipation region that follows a behavior as per $\mathrm{n}^{-7}$. The $\mathrm{n}^{-1}$ Tchen law appears clearly that related to an important turbulent production.

\section{REFERENCES}

1. So, R.M.C. and H. Aksoy, 1993. On vertical turbulent buoyant jets. Intl. J. Heat Mass Transfer, 36: 3187-3200.

2. Dai, Z., L.K. Tseng and G.M. Faeth, 1994. Structure of round, fully developed, buoyant turbulent plumes. J. Heat Transfer, 116: 409-417.

3. Shabbir, A. and W.K. George, 1994. Experiments in a round turbulent buoyant plume. J. Fluid Mech., 275: 1-32.

4. Brahimi, M. and D. Kim-Son, 1986. Experimental and numerical predictions of the mean flow of a turbulent pure plume. Arch. Mech. Warszawa, 38: 519-528.
5. Dai, Z., L.K. Tseng and G.M. Faeth, 1995. Velocity statistics of round, fully developed, buoyant turbulent plumes. J. Heat Transfer, 117: 138-145.

6. George, W.K., R.L. Alpert and F. Tamanini, 1977. Turbulence measurements in an axisymmetric buoyant plume. Intl. J. Heat Mass Transfer, 20: 1145-1154.

7. Mahmoud, A.O.M., R.B. Maad and A. Belghith, 1998. Interaction d'un écoulement de thermosiphon avec un panache thermique à symétrie axiale: étude expérimentale. Rev. Gén. Therm., 37: 385-396.

8. Zinoubi, J., 2003. Etude de l'interaction d'un écoulement de thermosiphon avec un panache thermique à symétrie axiale:Influence de paramètre de forme. Thèse de Doctorat, Université Elmanar, Faculté des Sciences de Tunis.

9. Zinoubi, J., R.B. Maad and A. Belghith, 2004. Influence of the vertical source-cylinder spacing on the interaction of thermal plume with a thermosiphon flow: An experimental study. Exp Therm. Fluid. Sci., 28: 329-336.

10. Zinoubi, J., A.O.M. Mahmoud., T. Naffouti., R.B. Maad and A. Belghith, 2006. Study of the flow structure of a thermal plume evolving in an unlimited and in a semi-enclosed environment. Am. J. Appl. Sci., 31: 1690-1697.

11. Pera, L. And B. Gebhart, 1975. Laminar plume interactions. J. Fluid Mech., 68: 259-271.

12. Agator, J.M., 1983. Contribution à l'étude de la structure turbulente d'un panache thermique à symétrie axiale. Interaction du panache avec son environnement limité, Thèse, Université de Poitiers.

13. Brahimi, M., M. Lamour and D. Kim-Son, 1988. Champs moyens et fluctuants des panaches thermiques isolés ou en interaction. Rev. Gen. Therm., 315-316: 236-243.

14. Brahimi, M., L. Dehmani and D. Kim-Son., 1989. Structure turbulente de l'écoulement d'interaction de deux panaches thermiques. Intl. J. Heat Mass Transfer, 32: 1551-1559.

15. Kim-Son, D., 1977. Contribution à l'étude de la zone de transition et de la zone de turbulence établie dans un écoulement de convection naturelle sur une plaque plane verticale isotherme. Thèse de Doctorat d'Etat, Université de Poitiers.

16. Lumley, J.L., 1965. The spectrum of nearly inertial turbulence in a stably stratified fluid. J. Atmos. Sci., 21: 99-102.

17. Kotsovinos, N., 1991. Turbulence spectra in free convection flow. Phys. Fluids A, 3: 163-167.

18. Sangras, R., Z. Dai and G.M. Faeth, 1988. Mixing structure of plane self-preserving buoyant turbulent plumes. ASME J. Heat Transfer, 120: 1033-1041.

19. Corrsin, S., 1951. On the spectrum of isotropic temperature fluctuations in an isotropic turbulence. J. Appl. Phys., 22: 469-473. 